\title{
DOK7V1 influences the malignant phenotype of lung cancer cells through PI3K/AKT/mTOR and FAK/paxillin signaling pathways
}

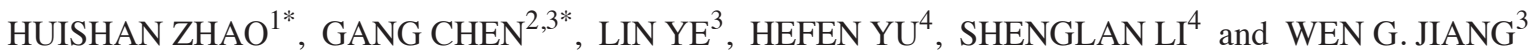 \\ ${ }^{1}$ Reproductive Medicine Centre, The Affiliated Yantai Yuhuangding Hospital of Qingdao University, Yantai, Shandong 264000; \\ ${ }^{2}$ Comprehensive Liver Cancer Center, Beijing 302 Hospital, Beijing 100039, P.R. China; ${ }^{3}$ Cardiff-China Medical Research \\ Collaborative, Cardiff University School of Medicine, Cardiff CF14 4XN, UK; ${ }^{4}$ Department of Biochemistry and \\ Molecular Biology, School of Basic Medical Sciences, Capital Medical University, Beijing 100069, P.R. China
}

Received June 28, 2018; Accepted October 19, 2018

DOI: $10.3892 /$ ijo.2018.4624

\begin{abstract}
Downstream of tyrosine kinase 7 transcript variant 1 (DOK7V1) is a docking protein mediating signal transduction between receptors and intracellular downstream molecules. Our previous study indicated that DOK7V1 was decreased in lung cancer and its lower expression was associated with a decreased survival rate. The 5-year overall survival rate for patients with lung cancer was 20.2 and $18.6 \%$ for high and low DOK7 expression, respectively; the 5-year disease-free survival rate for patients with lung cancer was 14.3 and $16.9 \%$ for high and low DOK7 expression, respectively. DOK7V1 inhibited proliferation and migration, but enhanced adhesion, of lung cancer cells. In the present study, the effect of DOK7V1 and its domains [pleckstrin homology $(\mathrm{PH})$ and phosphotyrosine-binding (PTB) domain] on the malignant phenotype and associated signaling pathway in lung cancer cells was investigated. The results indicated that truncation of DOK7V1 domains (DOK7V1 $\Delta-\mathrm{PH}$ and DOK7V1 $\Delta-\mathrm{PTB})$ inhibited the proliferation and migration of lung cancer cells which exhibited the same trend as DOK7V1, whereas DOK7V1 $\Delta-\mathrm{PH}$ and DOK7V1 $\Delta$-PTB exhibited different functions from those of DOK7V1 in cell matrix adhesion. Consistently, DOK7V1 overexpression in lung cancer cells suppressed the phosphoinositide 3-kinase (PI3K)/protein kinase B (AKT)/mammalian target of rapamycin (mTOR) signaling pathways, but activated the focal adhesion kinase $(\mathrm{FAK}) /$ paxillin signaling pathway. Taken together, these results indicate that DOK7V1 may inhibit proliferation and
\end{abstract}

Correspondence to: Dr Wen G. Jiang, Cardiff-China Medical Research Collaborative, Cardiff University School of Medicine, Heath Park, Cardiff CF14 4XN, UK

E-mail: jiangw@cardiff.ac.uk

*Contributed equally

Key words: downstream of tyrosine kinase 7, phosphotyrosinebinding domain, pleckstrin homology domain, proliferation, AKT, FAK migration via negatively regulating the $\mathrm{PI} 3 \mathrm{~K} / \mathrm{AKT} / \mathrm{mTOR}$ signaling pathway, and increase adhesion by upregulating the FAK/paxillin signaling pathway in lung cancer cells.

\section{Introduction}

Lung cancer remains the leading cause of cancer mortality and has become an increasingly serious public health burden globally (1). Lung cancer is categorized into small cell lung cancer (SCLC) and non-SCLC (NSCLC) following pathological pattern. NSCLC accounts for $\sim 80 \%$ of all lung cancer cases, including squamous cell carcinoma, adenocarcinoma, large cell carcinoma and adenosquamous cell carcinoma (2). NSCLC is often diagnosed at an advanced stage and the 5 -year survival rate remains $\sim 15 \%$ (3). Therefore, there is an urgent requirement to identify and screen for tumor-associated genes, further investigate the molecular mechanisms of NSCLC tumorigenesis and development, and identify novel biomarkers for early diagnosis and therapeutic targets of NSCLC. Tumor-associated genes rely on diverse signaling pathways to influence the proliferation, migration and invasion of tumor cells, and subsequently disseminate from primary tumors to metastasize through lymphatic and blood vessels. Understanding the molecular mechanisms underlying tumor metastasis has a critical significance in formulating treatment and early diagnosis. Receptor tyrosine kinases (RTKs) serve a vital function in regulating cellular process, including cell migration, proliferation, differentiation, survival and cell cycle regulation $(4,5)$. Deregulation of RTKs includes chromosomal translocations, autocrine activation, overexpression of RTKs or gain-of-function mutations which occurs in cancer (6). The downstream of tyrosine kinase (DOK) protein family consists of seven members, mediating intracellular signaling transduction downstream of RTKs (7-9). DOK proteins share a topology domain characterized by a central phosphotyrosine-binding (PTB) domain, an N-terminal pleckstrin homology $(\mathrm{PH})$ domain and Src homology 2 target motifs in the C-terminus $(7,10)$. On the basis of their location, DOK proteins are divided into three subgroups: DOKs 1-3 are mainly expressed in hematopoietic tissues (11), DOKs 4-6 are primarily present within the nervous system $(12,13)$, and DOK7 is predominantly in skeletal muscle and the heart, which 
exhibits a distinct function compared with other members (14). Aberrant expression of DOK proteins has been identified in multiple malignancies. DOKs 1-3 co-operatively suppress aggressive histiocytic sarcoma (11). DOK2 was regarded as a biomarker of patients with gastric cancer with poor prognosis $(15,16)$. In a mouse model, deletion of DOKs 1-3 resulted in abnormal proliferation of bronchoalveolar stem cells and alveolar type II cells, which subsequently progressed to lung cancer (7). In addition, a decreased expression level of DOK2 was observed in human lung cancer tissues, and forced DOK2 overexpression repressed proliferation of lung cancer cells (7).

DOK7, as a non-catalytic adaptor protein, is essential for the activation of the tyrosine kinase muscle-specific kinase (MuSK) and acetylcholine receptor clustering, which are indispensable for neuromuscular junctions $(17,18)$. The function of DOK7 in cancer was revealed gradually. Demethylation of DOK7 decreased the proliferation and invasion of esophageal squamous cell carcinoma cells (19). The PTB domain is the region through which DOK7 interacts with the juxtamembrane region of MuSK, although the PTB and PH domains are required for the activation of MuSK $(18,20,21)$. The PH domain consists of $\sim 120$ amino acids, mediating intracellular and extracellular signaling, also acting as important constituents of the cytoskeleton (22-24). PTB was also able to contact the cell membrane, and regulate intracellular and extracellular signaling. For example, PH and PTB domains of DOK4 are required for DOK4 localization at the membrane (10). The DOK1 PH domain appears to be required for tyrosine phosphorylation of the protein and its normal localization to a subcellular membrane component (10).

In our previous study, we identified that DOK7 transcripts were decreased in lung cancer, and the lower DOK7 level was associated with poorer overall survival and progression-free survival (25). Overexpression of the DOK 7 transcript variant 1 (DOK7V1) limited the proliferation and migration, but enhanced the adhesion to the extracellular matrix, of lung cancer cells (25). However, the underlying molecular mechanism of DOK7V1 and the functions of its domains in lung cancer cells remain unknown. Therefore, the aim of the present study was to investigate the functions of its domains and the molecular mechanism underlying its involvement in lung cancer cells.

\section{Materials and methods}

Cell culture. Lung cancer A549, SKMES-1 and H3122 cell lines were purchased from the American Type Culture Collection (Manassas, VA, USA). A549 and SKMES-1 cells were routinely cultured in Dulbecco's modified Eagle's medium (Thermo Fisher Scientific, Inc., Waltham, MA, USA) and H3122 cells were cultured in RPMI-1640 medium (Thermo Fisher Scientific, Inc.), supplemented with $10 \%$ fetal bovine serum (Shanghai ExCellBio, Inc., Shanghai, China), in an incubator at $37^{\circ} \mathrm{C}$ with a $95 \%$ humidified atmosphere containing $5 \% \mathrm{CO}_{2}$.

Construction of DOK7V1 plasmid and transfection. DOK7V1-Flag and pEnter (Vector) plasmids were purchased from Vigene Biosciences, Inc. (Rockville, MD, USA). DOK7V1 $\Delta-\mathrm{PH}$ and DOK7V1 $\Delta$-PTB were constructed using a
Q5 ${ }^{\circledR}$ Site-Directed Mutagenesis kit (Without Competent Cells) (New England BioLabs, Inc., Ipswich, MA, USA). Purified DOK7V1 and control plasmids were transfected into A549 and SKMES-1 cells using an Easyject Plus electroporator (EquiBio Ltd., Altrincham, UK). H3122 cells were transfected with DOK7V1, DOK7V1 $\Delta-\mathrm{PH}$ and DOK7V1 $\Delta$-PTB, respectively, using Neofect ${ }^{\mathrm{TM}}$ DNA transfection reagent (Neofectbiotech Co., Ltd., Beijing, China).

RNA isolation and reverse transcription-polymerase chain reaction ( $R T-P C R)$. Total RNA was isolated from A549, SKMES-1 and H3122 cells using TRIzol ${ }^{\circledR}$ reagent (Invitrogen; Thermo Fisher Scientific, Inc.). cDNA was generated from each $1 \mu \mathrm{g}$ RNA sample using a QuantiTect Reverse Transcription kit (Qiagen GmbH, Hilden, Germany), according to the manufacturer's protocol. PCR was performed as described previously (26) with the following primer sequences: DOK7, 5'-ACTGGGCTGGCGTCTTCTTCC-3' (forward) and 5'-TCGGACGATGCAGTCGAACAG-3' (reverse); and GAPDH, 5'-GGCTGCTTTTAACTCTGGTA-3' (forward) and 5'-GACTGTGGTCATGAGTCCTT-3' (reverse).

Western blot analysis. Cells were collected and lysed using Radioimmunoprecipitation Assay Lysis and Extraction Buffer (Thermo Fisher Scientific, Inc.), followed by centrifugation at $13,000 \mathrm{x} \mathrm{g}$ for $15 \mathrm{~min}$ at $4^{\circ} \mathrm{C}$. Total protein concentrations in the supernatant were determined using a DC Protein Assay kit (Bio-Rad Laboratories, Inc., Hercules, CA, USA) and an ELx800 spectrophotometer (Bio-Tek Instruments, Inc., Winooski, VT, USA). Equal amounts $(30 \mu \mathrm{g})$ of protein samples were separated by SDS-PAGE (10\% gel) and blotted onto a nitrocellulose membrane. The membrane was blocked using 5\% skimmed milk in Tris-buffered saline for $1 \mathrm{~h}$ at room temperature. Proteins were then probed using anti-human FLAG (1:1,000; cat. no. F3040; Sigma; Merck KGaA, Darmstadt, Germany), anti-DOK7 antibody (1:1,000; cat. no. ab75049; Abcam, Cambridge, MA, USA), anti-protein kinase B (AKT) antibody (1:1,000; cat. no. 2920; Cell Signaling Technology, Inc., Danvers, MA, USA), anti-phospho (p)-AKT (1:1,000; cat. no. 4051; Cell Signaling Technology, Inc.), anti-phosphoinositide 3-kinase (PI3K) antibody (1:500; cat. no. sc-365290; Santa Cruz Biotechnology, Inc., Dallas, TX, USA), anti-p-PI3K antibody (1:500; cat. no. sc-374534; Santa Cruz Biotechnology, Inc.), anti-mammalian target of rapamycin (mTOR) antibody (1:1,000; cat. no. ab2732; Abcam), anti-p-mTOR antibody (1:1,000; cat. no. 2971; Cell Signaling Technology, Inc.), anti-Rho-associated protein kinase (Rock) antibody (1:500; cat. no. sc-17794; Santa Cruz Biotechnology, Inc.), anti-p-Rock antibody (1:1,000; cat. no. ab2732; Abcam), anti-focal adhesion kinase (FAK) antibody (1:500; cat. no. sc-1688; Santa Cruz Biotechnology, Inc.), anti-p-FAK antibody (1:500; cat. no. sc-11766; Santa Cruz Biotechnology, Inc.), anti-paxillin antibody (1:500; cat. no. sc-365174; Santa Cruz Biotechnology, Inc.), anti-p-paxillin antibody (1:500; cat. no. sc-365020; Santa Cruz Biotechnology, Inc.), anti-human GAPDH antibody (1:500; cat. no. sc-47724; Santa Cruz Biotechnology, Inc.) and corresponding horseradish peroxidase (HRP)-conjugated goat anti-rabbit and goat anti-mouse immunoglobulin $\mathrm{G}$ secondary antibodies (1:3,000; cat. nos. ZB-2301M and ZB-2305; OriGene Technologies, Inc., 
A

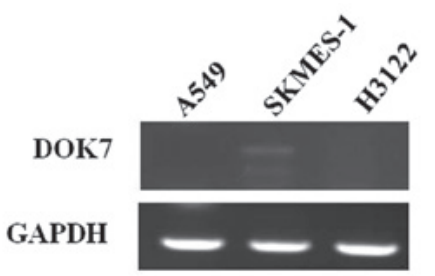

B
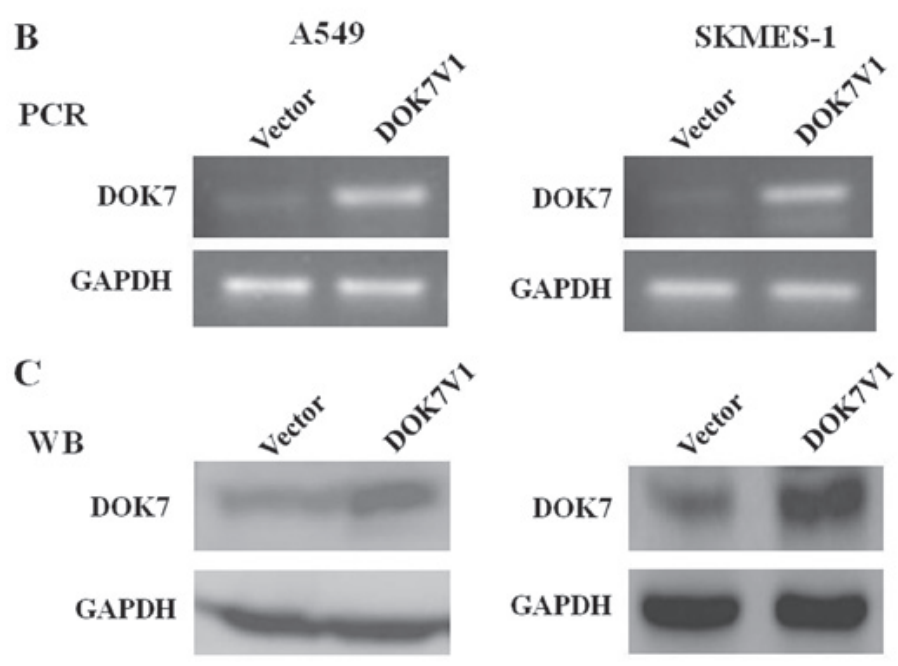

Figure 1. Expression level of DOK7V1 in lung cancer cell lines. (A) Expression of DOK7V1 in A549, SKMES-1 and H3122 cells was determined using RT-PCR analysis. Following transfection, respective overexpression of DOK7V1 in A549 and SKMES-1 cells were determined using (B) RT-PCR and (C) western blot analysis. DOK7V1, downstream of tyrosine kinase 7 transcript variant 1; RT-PCR, reverse transcription-polymerase chain reaction; WB, western blot.

Beijing, China), respectively. Protein bands were visualized and analyzed using Luminata Forte western blot HRP substrate (Merck KGaA) and a UV imager (Uvitec, Inc., Cambridge, UK).

Immunofluorescence. Cells on glass coverslips were rinsed with PBS three times, and then fixed with $4 \%$ paraformaldehyde for $20 \mathrm{~min}$ at room temperature. Anti-human FLAG was diluted $1: 1,000$ in blocking buffer (1\% bovine serum albumin in PBS), prior to adding to the coverslips and incubating for $1 \mathrm{~h}$ at room temperature. Following three washes with PBS, the coverslips were incubated using Alexa Fluor ${ }^{\circledR}$ 488-conjugated secondary antibodies (1:100; cat. no. A-21205; Thermo Fisher Scientific, Inc.) for $45 \mathrm{~min}$ at room temperature. Following three further washes with PBS, nuclei were stained with DAPI (1:500,000; cat. no. D-1306; Thermo Fisher Scientific, Inc.) for $2 \mathrm{~min}$ at room temperature, followed by three further washes with PBS. The coverslips were mounted on glass slides and incubated at room temperature overnight. Immunofluorescence images were visualized using a confocal microscopy system (Leica TCS SP8; Leica Microsystems GmbH, Wetzlar, Germany) with a $63 \mathrm{X}$ oil-immersion objective.

In vitro cell proliferation assays. A total of 3,000 cells in $200 \mu \mathrm{l}$ culture medium was added to each well of a 96-well plate. A total of five plates were used to obtain cell density readings following incubation at $37^{\circ} \mathrm{C}$ for up to 4 days. Following incubation, the medium was removed and $100 \mu 1$ 10\% Cell Counting Kit-8 (Dojindo Molecular Technologies, Inc., Kumamoto, Japan) was added for $1 \mathrm{~h}$ at $37^{\circ} \mathrm{C}$. Subsequently, the absorbance was determined at a wavelength of $450 \mathrm{~nm}$ using a spectrophotometer (BioTek Instruments, Inc.).
In vitro tumor cell migration assay. Cells ( $1 \times 10^{6}$ cells/well) were seeded in a 6 -well plate and cultured in an incubator at $37^{\circ} \mathrm{C}$ overnight. The cell monolayer was scratched with a $200-\mu 1$ pipette tip to create an artificial wound prior to washing twice with PBS to remove floating cells. The migration of cells was monitored and recorded every $6 \mathrm{~h}$ using an inverted light microscope at x10 and x 20 magnification for $24 \mathrm{~h}$. The wound distance was determined and analyzed using ImageJ software (version 1.62; National Institutes of Health, Bethesda, MD, USA).

In vitro tumor cell Matrigel adhesion assay. A 96-well plate was coated with $5 \mu \mathrm{g}$ Matrigel/100 $\mu \mathrm{l} /$ well and air-dried overnight at room temperature. Following rehydration, 20,000 cells $/ 200 \mu \mathrm{l} /$ well were seeded into each well for 40 min. Following incubation, medium was removed and the plate was washed three times with PBS to remove the non-adherent cells. Adherent cells were fixed with $4 \%$ formaldehyde for $30 \mathrm{~min}$ prior to being stained with $0.5 \%$ crystal violet solution for $10 \mathrm{~min}$ at room temperature. Subsequently, crystal violet was dissolved in $10 \%$ acetic acid and the absorbance at $570 \mathrm{~nm}$ was determined using a spectrophotometer (BioTek Instruments, Inc.).

Gene set enrichment analysis (GSEA). The association between biological processes/pathway, phenotypes and DOK7 expression level was analyzed using the GSEA program (version 2.2; www.broad.mit.edu/gsea). Samples from The Cancer Genome Atlas (TCGA) datasets were separated into high or low DOK7 expression groups using the median as the cut-off. GSEA was used to calculate a pathway Enrichment Score that assessed whether genes from a predefined gene set 


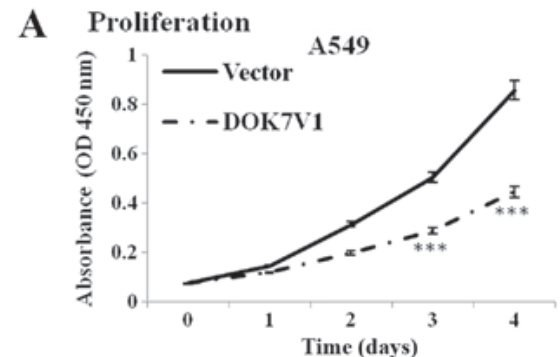

B Migration

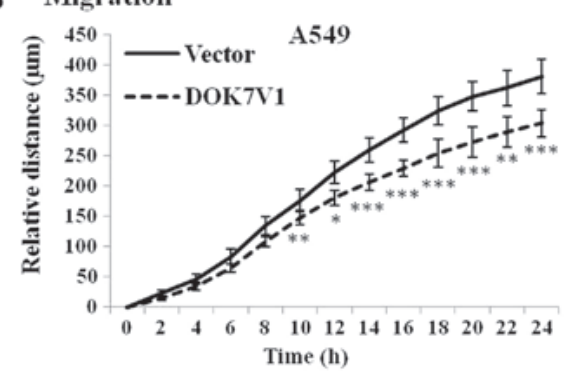

C Adhesion

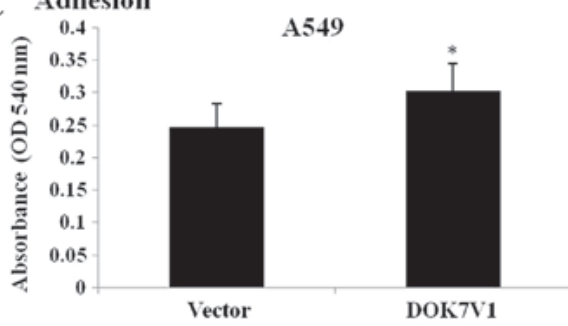

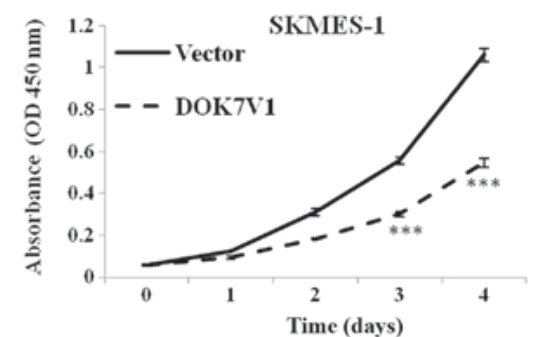
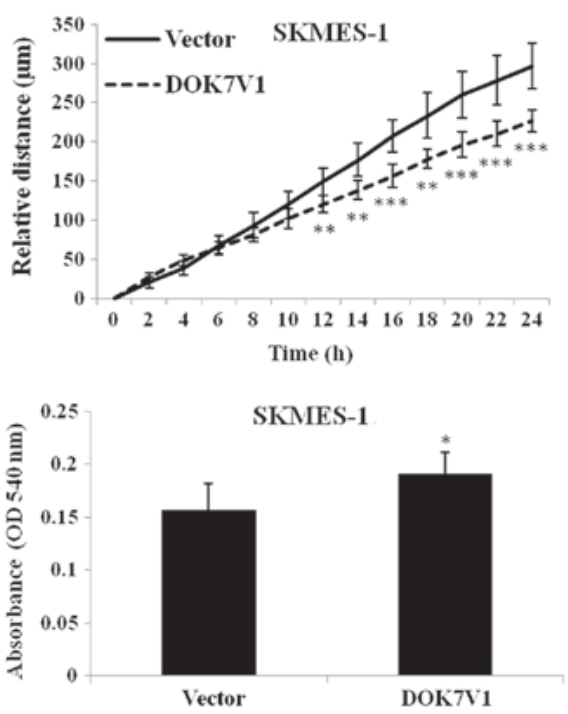

Figure 2. Effect of forced DOK7V1 expression on biological functions in lung cancer cells. (A) Increased DOK7V1 expression inhibited the in vitro proliferation of A549 and SKMES-1 cells. (B) DOK7V1 overexpression in A549 and SKMES-1 cells exhibited an inhibitory effect on cell motility. (C) Expression of DOK7V1 increased cell-matrix adhesion of lung cancer cells. Results are representative of three independent experiments for each function assay. "P<0.05, ${ }^{* *} \mathrm{P}<0.01,{ }^{* * * *} \mathrm{P}<0.001$ vs. vector control. DOK7V1, downstream of tyrosine kinase 7 transcript variant 1 ; OD, optical density.

of PI3K/AKT/mTOR and FAK/paxillin signaling pathways were enriched among the high- (or low-) ranked genes or distributed randomly. Default settings were utilized when using software applications. Significance was determined by permutation analysis (1,000 permutations) and calculation of the false discovery rate (FDR). A gene set was considered to be significantly enriched when the FDR score was $<0.05$.

Statistical analysis. Data are presented as the mean \pm standard deviation. Experimental procedures were repeated independently at least three times. Statistical analysis was performed using a two-sided Student's t-test for two-group comparisons and by one-way ANOVA, followed by a Bonferroni post hoc test, for multiple group comparisons. All statistical analyses were performed using Prism (version 5; GraphPad Software, Inc., La Jolla, CA, USA). P $<0.05$ was considered to indicate a statistically significant difference.

\section{Results}

Overexpression of DOK7V1 has an inhibitory effect on proliferation and migration, but a positive effect on adhesion in lung cancer cells. To select the optimal cell lines for further investigation, the expression of DOK7V1 in A549, SKMES-1 and $\mathrm{H} 3122$ cells was determined using RT-PCR analysis. The stable transfection of DOK7V1 in the lung cancer cell lines was verified by RT-PCR and western blot analysis (Fig. 1). To further confirm the effect of DOK7V1 on malignant phenotypes of lung cancer cells, the experiments were performed in A549 and SKMES-1 cells. The results indicated that overexpression of DOK7V1 significantly decreased the proliferation and migration, but significantly increased the adhesion, of A549 and SKMES-1 cells (Fig. 2).

PH domain and PTB domain determined the distribution of DOK7V1 in membrane and cytoplasm. DOK7V1 contains two functional domains: A PH domain and a PTB domain. To investigate the structural determinant regulating the intracellular distribution and expression of DOK7V1, as well as the biological function, two DOK7V1 truncated fragments were constructed based on the wild-type protein, DOK7V1 $\Delta-\mathrm{PH}$ and DOK7V1 $\Delta$-PTB (Fig. 3A). The immunofluorescence imaging results indicated that wild-type DOK7V1 was located and expressed in the membrane and cytoplasm. The truncated versions of DOK7V1 without the two functional domains led to a shift in the localization of DOK7V1 protein from the cytoplasm to be perinuclear (Fig. 3B). The results indicated that these two functional domains determined the distribution of DOK7V1 in the membrane and cytoplasm, which may influence the function of DOK7V1 in lung cancer cells.

Effect of the PH domain and PTB domain of DOK7V1 on the proliferation, migration and adhesion of lung cancer cells. 
A
DOK7V1
\begin{tabular}{l|l|ll|}
\hline PH & PTB & YY & Y Y \\
\hline
\end{tabular}
$1515 \mathrm{bp}$
DOK7V1 $1-\mathrm{PH}$

\begin{tabular}{|l|ll|}
\hline PTB & Y Y Y Y \\
\hline
\end{tabular}
$1197 \mathrm{bp}$

DOK7V11-PTB
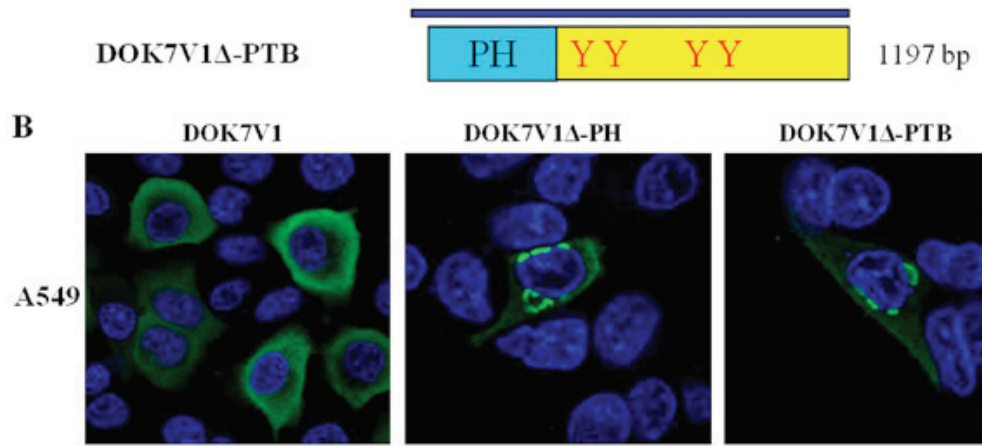

\section{DOK7V1A-PTB}

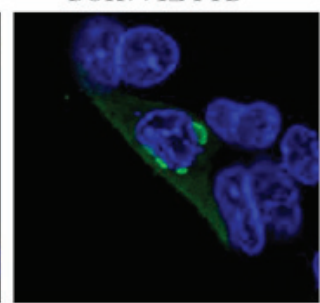

Figure 3. PH and PTB domains determine the distribution of DOK7V1 in the nuclear membrane and cytoplasm. (A) Schematic representation of DOK7V1 and its truncated fragments. (B) The cytoplasm was stained green and the nucleus was stained blue. Merged images are presented. The truncated versions of DOK7V1 without the PH or PTB functional domain appeared to have an effect on the localization of the protein. Intensive staining may be observed surrounding the nucleus. PH, pleckstrin homology; PTB, phosphotyrosine-binding; DOK7V1, downstream of tyrosine kinase 7 transcript variant 1.

A
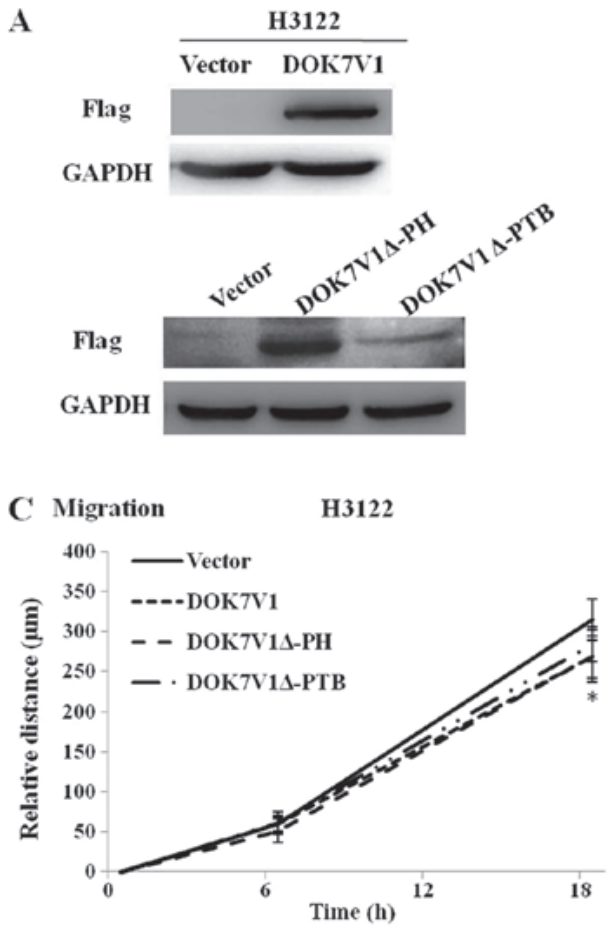

B

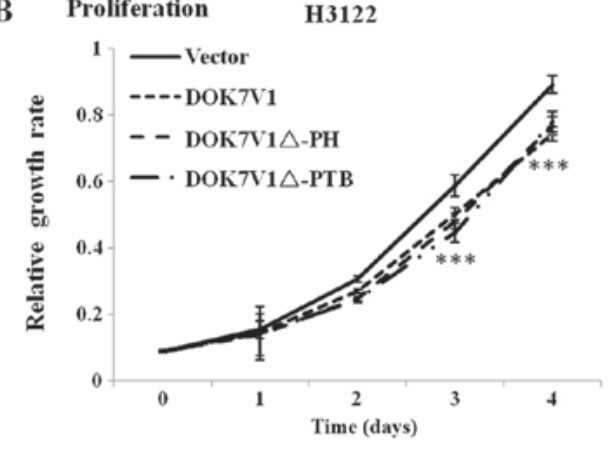

D Adhesion $\mathbf{H} 3122$

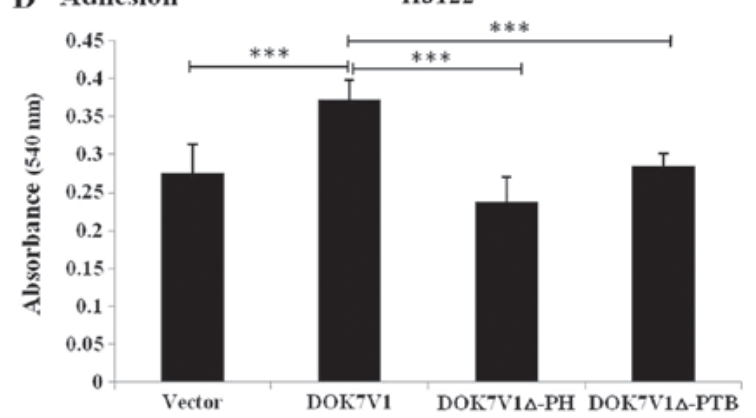

Figure 4. Effect of PH domain and PTB domain of DOK7V1 on lung cancer cell proliferation, migration and adhesion. (A) Wild-type and two truncated versions of DOK7V1 were stably transfected into H3122 cells and detected with an anti-FLAG antibody. (B) DOK7V1 $\Delta$-PH and DOK7V1 $\Delta$-PTB decreased the proliferation and migration (C), which was similar to that with DOK7V1. (D) DOK7V1 $\Delta$-PH and DOK7V1 $\Delta$-PTB truncated versions lost the adhesion-promoter effect observed in DOK7V1. "P<0.05, ${ }^{* * *} \mathrm{P}<0.001$ vs. vector control. PH, pleckstrin homology; PTB, phosphotyrosine-binding domain; DOK7V1, downstream of tyrosine kinase 7 transcript variant 1.

To further assess the biological function of DOK7V1 $\Delta-\mathrm{PH}$ and DOK7V1 $\Delta$-PTB, these truncated versions were stably transfected into $\mathrm{H} 3122$ cells and their expression was determined by western blotting. DOK7V1, DOK7V1 $\Delta-\mathrm{PH}$ and DOK7V1 $\Delta$-PTB were identified to exhibit increased expression in stably transfected cells compared with in the corresponding control cells (Fig. 4A). It was identified that cell proliferation was significantly decreased from the third day, and migration was significantly decreased at $18 \mathrm{~h}$, in cells singly overexpressing DOK7V1 $\Delta$-PH and DOK7V1 $\Delta$-PTB compared with vector control cells, which exhibited the same trend as DOK7V1 (Fig. 4B and 4C). The adhesion assay revealed an increased rate in DOK7V1-overexpressing cells compared with the control group; however, the DOK7V1 $\Delta-\mathrm{PH}$ and DOK7V1 $\triangle$-PTB truncated versions lost the tumor-promoting effects identified in DOK7V1 (Fig. 4D). 


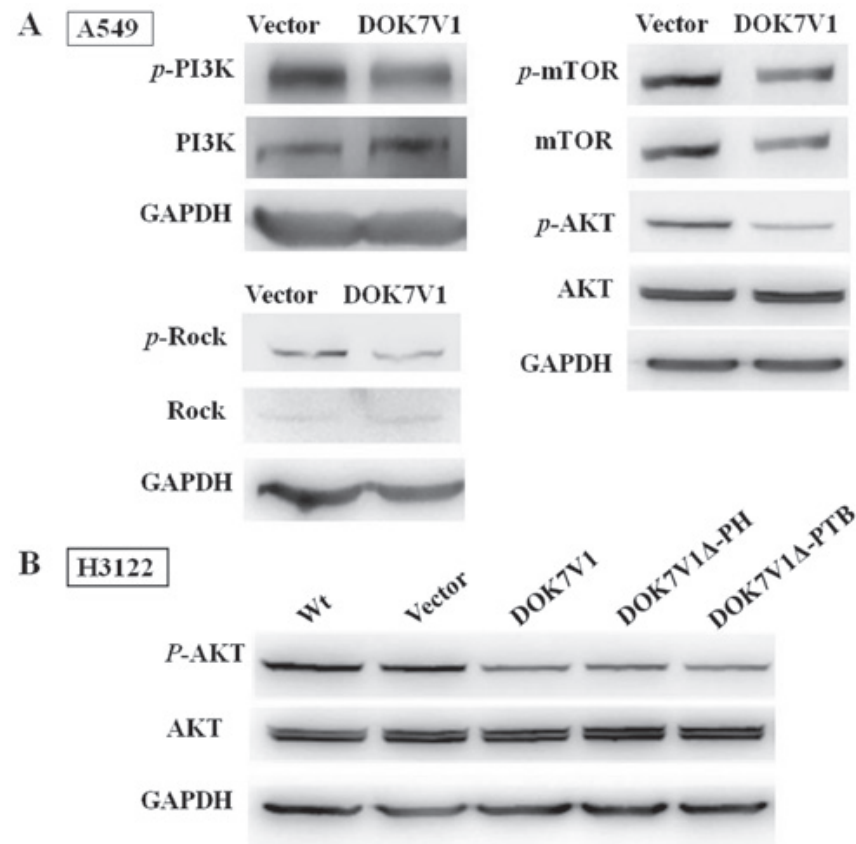

Figure 5. No differences exist between the two truncated versions and DOK7V1 in inhibiting PI3K/AKT/mTOR signal pathway phosphorylation. (A) Immunoblotting was performed using anti-p-AKT and anti-AKT, p-mTOR and mTOR, p-PI3K and PI3K, Rock and p-Rock. GAPDH, AKT and mTOR were separated in the same lane of the gel. (B) DOK7V1 $\Delta-\mathrm{PH}$ and DOK7V1 $\Delta$-PTB did not result in the loss-of-function of the DOK7V1-mediated inhibition of AKT activation. DOK7V1, downstream of tyrosine kinase 7 transcript variant 1; PI3K, phosphoinositide 3-kinase; AKT, protein kinase B; mTOR, mammalian target of rapamycin; p-, phospho-; Rock, Rho-associated protein kinase; PH, pleckstrin homology; PTB, phosphotyrosine-binding; Wt, wild-type (cells without treatment)

No differences exist between the two truncated versions and DOK7V1 in inhibiting PI3K/AKT/mTOR signal pathway phosphorylation. To further study the signal pathways in which DOK7V1 is involved, the protein levels and phosphorylation levels of AKT, PI3K, mTOR and Rock in A549 cells were determined. Decreased levels of AKT, PI3K and Rock phosphorylation were observed in A549 cells with DOK7V1 overexpression, whereas the total AKT, PI3K and Rock protein levels were similar in comparison with their corresponding controls (Fig. 5A). mTOR phosphorylation and the total mTOR protein levels were decreased in A549 cell lines with DOK7V1 overexpression (Fig. 5A). The AKT phosphorylation status was investigated in $\mathrm{H} 3122$ cells transfected with various DOK7V1 constructs. Wild-type DOK7V1 overexpression significantly arrested AKT activation, and this inhibition was similar in cells expressing the

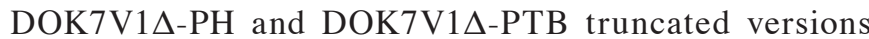
(Fig. 5B). Taken together, these results indicated that the two truncated version of DOK7V1 did not eliminate the inhibitory function of DOK7V1 in activating AKT.

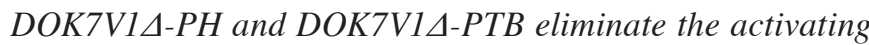
effect of DOK7VI on FAK and paxillin. FAK and paxillin are important proteins in focal adhesion, by connecting the cytoskeleton to the extracellular matrix. In view of DOK7V1 increasing the adhesion of lung cancer cells, the changes in associated factors in the FAK/paxillin signaling pathway were investigated. The overexpression of DOK7V1 significantly increased FAK and paxillin phosphorylation (Fig. 6A) in A549 cells; however, this influence was partially eliminated in

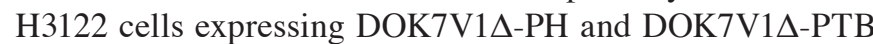
in cells (Fig. 6B). These results indicated that the $\mathrm{PH}$ domain and PTB domain serve a key function in regulating cell adhesion.

DOK7 overexpression is negatively associated with activation of the PI3K/AKT/mTOR signaling pathway and is positively associated with adherent ability. To further analyze the function of DOK7 expression in PI3K/AKT/mTOR signaling in lung cancer, GSEA was performed using a lung cancer dataset from TCGA. The lung cancer samples were separated according to the median DOK7 level in the specimens into high or low expression groups. Enrichment plots of GSEA demonstrated that the gene set of PI3K/AKT/mTOR signaling was primarily enriched in the low DOK7 expression group (Fig. 7A). The association between DOK7 expression and activation of FAK/paxillin signaling in lung cancer was identified using GSEA. As presented in Fig. 7B, the gene signatures of adhesion, which emphasized the importance of FAK in its description, were enriched in patients with high DOK7 expression. These results further suggested that, in lung cancer samples, DOK7 expression was negatively associated with $\mathrm{PI} 3 \mathrm{~K} / \mathrm{AKT} / \mathrm{mTOR}$ signaling activation and is positively associated with activation of the FAK/paxillin signaling pathway.

\section{Discussion}

In our previous study, we preliminarily studied the function of DOK7V1 in lung cancer cells and the association with the prognosis of patients with lung cancer (25). In the present study, the focus was on the mechanisms and functions of DOK7V1 and its domains in the lung cancer cells. The results of the present study suggested a hitherto unknown regulatory mechanism of DOK7V1 in lung cancer cells, which influences 

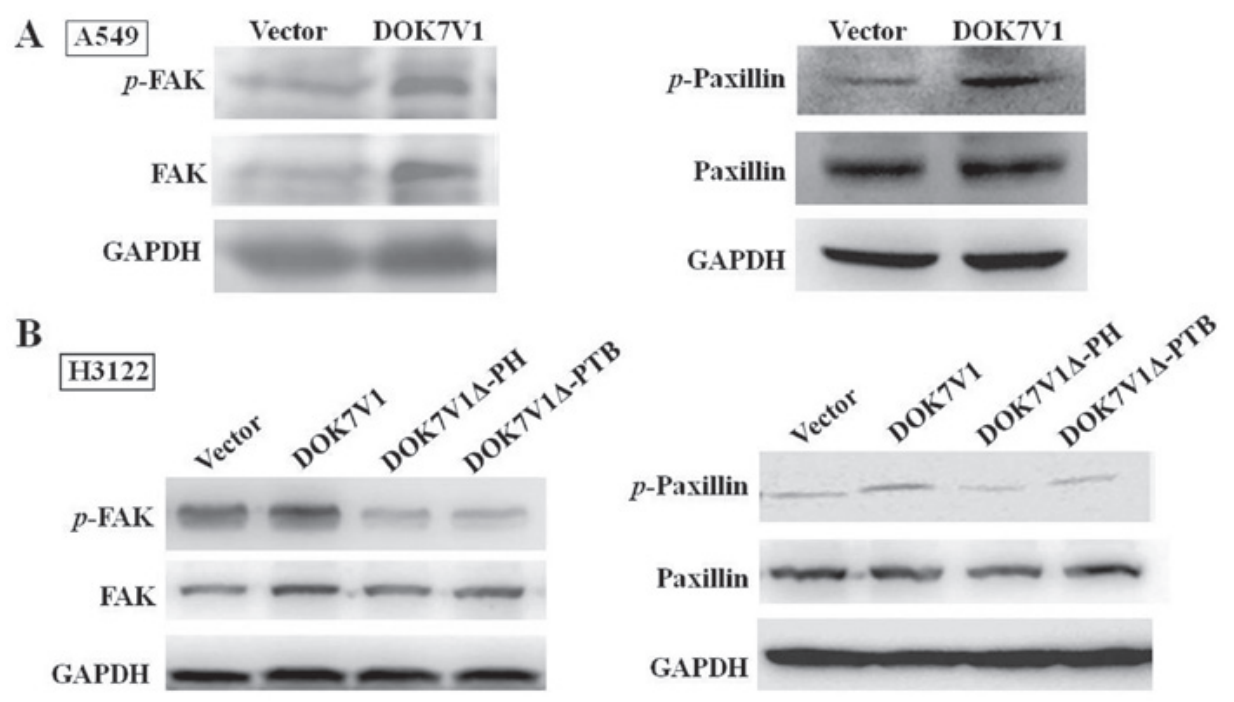

Figure 6. DOK7V1 $\triangle$-PH and DOK7V1 $\triangle$-PTB eliminate the activating effect of DOK7V1 on FAK and paxillin activation. (A) The phosphorylation of FAK and paxillin were activated when DOK7V1 was overexpressed in A549 cells. GAPDH, FAK and paxillin were separated in the same lane of the gel. (B) DOK7V1 $\triangle$-PH and DOK7V1 $\triangle$-PTB led to the loss of function of the DOK7V1-mediated promotion of FAK and paxillin activation. DOK7V1, downstream of tyrosine kinase 7 transcript variant 1; PH, pleckstrin homology; PTB, phosphotyrosine-binding; FAK, focal adhesion kinase; p-, phospho-
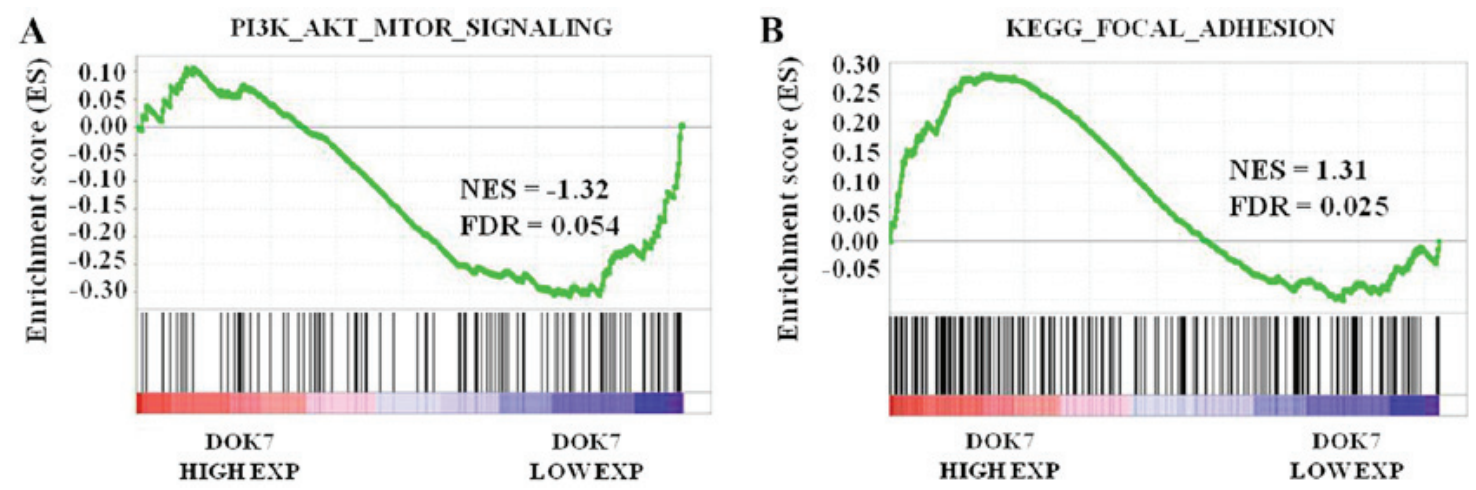

Figure 7. DOK7 expression is negatively associated with activation of the PI3K/AKT/mTOR signaling pathway, but is positively associated with activation of the FAK/paxillin signaling pathway. (A) DOK7 enrichment plots of gene expression signatures for the PI3K/AKT/mTOR signaling pathway according to DOK7 mRNA expression levels by gene set enrichment analysis of samples from The Cancer Genome Atlas. (B) The gene signatures of adhesion associated with FAK were enriched in patients with high DOK7 expression. DOK7, downstream of tyrosine kinase 7; PI3K, phosphoinositide 3-kinase; AKT, protein kinase B; mTOR, mammalian target of rapamycin; FAK, focal adhesion kinase; NES, normalized enrichment score; FDR, false discovery rate; EXP, expression.

cell adhesion, but not the proliferation and migration by its primary domain.

The functions of the DOK protein family in lung cancer have gradually been identified. DOKs 1-3 have been identified to be absent from or decreased in lung cancer (23). In mouse models, a lack of DOKs 1-3 promoted the proliferation of bronchoalveolar stem cells and alveolar type II cells, which resulted in the development of lung cancer, particularly in triple knockout mouse models (11). Decreased DOK2 levels were identified in primary lung adenocarcinomas and lymph node metastases, whereas no difference in expression of DOK1 and DOK3 was identified in lung cancer compared with normal tissues, although a decreased DOK3 level was observed in lymph node metastasis tissues when compared with its expression in primary tumor (7). DOK2 was identified as a tumor suppressor gene of lung cancer, since DOK2 was associated with epidermal growth factor receptor mutations to promote lung cancer (27). DOK7 was the primary focus in the present study. In breast cancer, DOK7 is downregulated owing to the hypermethylation of its $\mathrm{CpG}$ islands (22). The results of this study indicated that DOK proteins may serve an inhibitory function in cancer development, and gene deletion or hypermethylation of DOK proteins may occur in cancer cells. In addition, our previous study identified a lower expression level of DOK7 in lung cancer and DOK7V1 overexpression, resulting in a decrease in proliferation and migration, and an increase in adhesion in lung cancer cells (25). In the present study, the experiments in which DOK7V1 inhibited proliferation and migration, but promoted adhesion in other lung cancer cell lines were repeated to confirm the effect of DOK7V1 on the malignant phenotype. Furthermore, the change of invasion following transfection of lung cancer cells with DOK7V1 was investigated, but no difference between the test group and control group was identified.

DOK7V1 is a 1,515-residue protein, containing a $\mathrm{PH}$ domain, a PTB domain and C-terminal sites of tyrosine 
phosphorylation. DOK7 is a substrate of MuSK and also an activator of its kinase activity, and is therefore an important protein for forming the vertebrate neuromuscular junction, and may facilitate trans-autophosphorylation of the kinase activation loop via a dimeric arrangement of its $\mathrm{PH}$ and PTB domains (28). A truncation of the domains may lead to changes in protein localization. A recent study revealed that DOK7 bound to membranes containing phosphatidylinositol phosphates (PIPs) through PH domain binding, facilitating local clustering of PIP molecules in the bilayer (29). In the present study, the PH and PTB domains were identified to determine the localization of DOK7V1 in the membrane and cytoplasm, as revealed by the nuclear membrane distribution of PH- and PTB-truncated DOK7V1 proteins (DOK7V1 $\Delta-\mathrm{PH}$ and DOK7V1 $\Delta$-PTB). Does this change in location have an effect on the function of the protein? In the present study, in vitro functional experiments indicated that DOK7V1 overexpression inhibited $\mathrm{H} 3122$ cell proliferation, motility and adhesion, and DOK7V1 $\Delta-\mathrm{PH}$ and DOK7V1 $\Delta$-PTB have the same effect on proliferation and motility as DOK7V1, but with the enhancement of adhesion eliminated.

Dysregulation of the PI3K/AKT/mTOR signaling pathway has been implicated in the cancerous migration, proliferation and poor prognosis of various types of cancer, including lung cancer (30-32). Tumor suppressor genes decreased the proliferation and migration of NSCLC by inhibiting the $\mathrm{PI} 3 \mathrm{~K} / \mathrm{AKT} / \mathrm{mTOR}$ signaling pathway (33). The in vitro results of the present study indicated that DOK7V1 inhibited the phosphorylation of AKT, PI3K, mTOR and ROCK in A549 cells. The two truncated versions of DOK7V1 also could not active AKT in H3122 cells. PI3K/AKT signaling appears to rely on DOK7 in the cytoplasm or associated with the RTK. The weakened PI3K/AKT signaling may be caused by the retention of truncated DOK7V1 at the perinuclear area. Further analysis indicated that an increased level of DOK7V1 was negatively associated with the activation of PI3K/AKT/mTOR signaling. According to these results, we hypothesize that DOK7V1 overexpression may inhibit the proliferation and migration by suppressing the activation of PI3K/AKT/mTOR signaling pathway. However, a clear conclusion cannot be made. Investigation of whether DOK7V1 decreases the proliferation and migration of lung cancer cells following inhibition of the PI3K/AKT/mTOR signaling pathway is required in future studies.

Hyperactivation of the FAK/paxillin signaling pathway was identified to be significantly associated with cell adhesion (34-36). FAK and paxillin dynamics serve an essential function in regulating the adhesion of various cells (37). In the present study, an increased adhesion rate of cells overexpressing DOK7V1 compared with that of the control group was revealed; however, the DOK7V1 $\Delta-\mathrm{PH}$ and DOK7V1 $\Delta$-PTB truncated versions eliminated the tumor-promoting effects observed in DOK7V1. Conversely, FAK and paxillin were also weakened, which provides further evidence of the association between its location and function. Furthermore, it was confirmed that the gene signatures associated with FAK/paxillin signaling activation were enriched in patients with high DOK7 expression. These results further suggested that in lung cancer specimens, DOK7 expression was positively associated with activation of the FAK/paxillin signaling pathway. Certainly, further research is required to reach a clear conclusion.
In summary, it was identified that DOK7V1 downregulation is associated with poor prognosis of patients with lung cancer. The truncations of the DOK7V1 domains appeared to have effect on the localization of the protein. DOK7V1 overexpression reversed the malignant phenotypes of H3122 cells, including proliferation, migration and adhesion. DOK7V1 $\Delta$-PH and DOK7V1 $\Delta$-PTB were able to eliminate the function of DOK7 in adhesion, but not in proliferation and migration. The two truncated version retained the inhibitory effect of DOK7V1 on AKT activation, but inactivated the enhanced effect of DOK7V1 on FAK and paxillin. We hypothesize that DOK7V1 may inhibit proliferation and migration via negatively regulating the PI3K/AKT/mTOR signaling pathway and increasing adhesion by upregulating the FAK/paxillin signaling pathway in lung cancer cells. The results of the present study provide a novel basis to improve our understanding of the pathogenesis of lung cancer.

\section{Acknowledgements}

Not applicable.

\section{Funding}

The present study was supported by Shandong Provincial Natural Science Foundation, China (grant no. ZR2017PH047) and the Research Foundation of Yantai Yuhuangding Hospital (grant no. 201604).

\section{Availability of data and materials}

The datasets used and/or analyzed during the present study are available from the corresponding author on reasonable request.

\section{Authors' contributions}

WGJ, GC, HZ and LY designed the study. GC, HY, LY and SL performed the experiments. $\mathrm{HZ}$ and GC analyzed the data, and prepared and revised the paper. All authors had final approval of the submitted and published versions of the paper.

\section{Ethics approval and consent to participate}

Not applicable.

\section{Patient consent for publication}

Not applicable.

\section{Competing interests}

The authors declare that they have no competing interests.

\section{References}

1. Siegel RL, Miller KD and Jemal A: Cancer Statistics, 2017. CA Cancer J Clin 67: 7-30, 2017.

2. Goldstraw P, Ball D, Jett JR, Le Chevalier T, Lim E, Nicholson AG and Shepherd FA: Non-small-cell lung cancer. Lancet 378: 1727-1740, 2011. 
3. Yang M, Lewinska M, Fan X, Zhu J and Yuan ZM: PRR14 is a novel activator of the PI3K pathway promoting lung carcinogenesis. Oncogene 35: 5527-5538, 2016.

4. Blume-Jensen P and Hunter T: Oncogenic kinase signalling. Nature 411: 355-365, 2001.

5. Ullrich A and Schlessinger J: Signal transduction by receptors with tyrosine kinase activity. Cell 61: 203-212, 1990.

6. Lemmon MA and Schlessinger J: Cell signaling by receptor tyrosine kinases. Cell 141: 1117-1134, 2010.

7. Berger AH, Niki M, Morotti A, Taylor BS, Socci ND, Viale A, Brennan C, Szoke J, Motoi N, Rothman PB, et al: Identification of DOK genes as lung tumor suppressors. Nat Genet 42: 216-223, 2010.

8. Simister PC and Feller SM: Order and disorder in large multi-site docking proteins of the Gab family--implications for signalling complex formation and inhibitor design strategies. Mol Biosyst 8: 33-46, 2012

9. Jones $\mathrm{N}$ and Dumont DJ: Recruitment of Dok-R to the EGF receptor through its PTB domain is required for attenuation of Erk MAP kinase activation. Curr Biol 9: 1057-1060, 1999.

10. Bedirian A, Baldwin C, Abe J, Takano T and Lemay S: Pleckstrin homology and phosphotyrosine-binding domain-dependent membrane association and tyrosine phosphorylation of Dok-4, an inhibitory adapter molecule expressed in epithelial cells. J Biol Chem 279: 19335-19349, 2004.

11. Mashima R, Honda K, Yang Y, Morita Y, Inoue A, Arimura S, Nishina H, Ema H, Nakauchi H, Seed B, et al: Mice lacking Dok-1, Dok-2, and Dok-3 succumb to aggressive histiocytic sarcoma. Lab Invest 90: 1357-1364, 2010.

12. Crowder RJ, Enomoto H, Yang M, Johnson EM Jr and Milbrandt J: Dok-6, a Novel p62 Dok family member, promotes Ret-mediated neurite outgrowth. J Biol Chem 279: 42072-42081, 2004.

13. Grimm J, Sachs M, Britsch S, Di Cesare S, Schwarz-Romond T, Alitalo K and Birchmeier W: Novel p62dok family members, dok-4 and dok-5, are substrates of the c-Ret receptor tyrosine kinase and mediate neuronal differentiation. J Cell Biol 154: 345-354, 2001

14. Cossins J, Liu WW, Belaya K, Maxwell S, Oldridge M, Lester T, Robb S and Beeson D: The spectrum of mutations that underlie the neuromuscular junction synaptopathy in DOK7 congenital myasthenic syndrome. Hum Mol Genet 21: 3765-3775, 2012.

15. An CH, Kim MS, Yoo NJ and Lee SH: Mutational and expressional analysis of a haploinsufficient tumor suppressor gene DOK2 in gastric and colorectal cancers. APMIS 119: 562-564, 2011.

16. Miyagaki H, Yamasaki M, Takahashi T, Kurokawa Y, Miyata H, Nakajima K, Takiguchi S, Fujiwara Y, Mori M and Doki Y: DOK2 as a marker of poor prognosis of patients with gastric adenocarcinoma after curative resection. Ann Surg Oncol 19: $1560-1567,2012$

17. Yamanashi Y, Higuch O and Beeson D: Dok-7/MuSK signaling and a congenital myasthenic syndrome. Acta Myol 27: 25-29, 2008.

18. 18. Okada K, Inoue A, Okada M, Murata Y, Kakuta S, Jigami T, Kubo S, Shiraishi H, Eguchi K, Motomura M, et al: The muscle protein Dok-7 is essential for neuromuscular synaptogenesis. Science 312: 1802-1805, 2006.

19. Yang SM, Li SY, Yu HB, Li JR and Sun LL: Repression of DOK7 mediated by DNMT3A promotes the proliferation and invasion of KYSE410 and TE-12 ESCC cells. Biomed Pharmacother 90: 93-99, 2017.

20. Hamuro J, Higuchi O, Okada K, Ueno M, Iemura S, Natsume T, Spearman H, Beeson D and Yamanashi Y: Mutations causing DOK7 congenital myasthenia ablate functional motifs in Dok-7. J Biol Chem 283: 5518-5524, 2008.
21. Hallock PT, Xu CF, Park TJ, Neubert TA, Curran T and Burden SJ: Dok-7 regulates neuromuscular synapse formation by recruiting Crk and Crk-L. Genes Dev 24: 2451-2461, 2010.

22. Mayer BJ, Ren R, Clark KL and Baltimore D: A putative modular domain present in diverse signaling proteins. Cell 73: 629-630, 1993.

23. Pawson T: Protein modules and signalling networks. Nature 373 : 573-580, 1995

24. Saraste M and Hyvönen M: Pleckstrin homology domains: A fact file. Curr Opin Struct Biol 5: 403-408, 1995.

25. Chen G, Yu H, Satherley L, Zabkiewicz C, Resaul J, Zhao H, Mu H, Zhi X, He J, Ye L, et al: The downstream of tyrosine kinase 7 is reduced in lung cancer and is associated with poor survival of patients with lung cancer. Oncol Rep 37: 2695-2701, 2017.

26. Zhao H, Yu H, Martin TA, Zhang Y, Chen G and Jiang WG: Effect of junctional adhesion molecule-2 expression on cell growth, invasion and migration in human colorectal cancer. Int $\mathrm{J}$ Oncol 48: 929-936, 2016.

27. Berger AH, Chen M, Morotti A, Janas JA, Niki M, Bronson RT, Taylor BS, Ladanyi M, Van Aelst L, Politi K, et al: DOK2 inhibits EGFR-mutated lung adenocarcinoma. PLoS One 8: e79526, 2013.

28. Bergamin E, Hallock PT, Burden SJ and Hubbard SR: The cytoplasmic adaptor protein Dok7 activates the receptor tyrosine kinase MuSK via dimerization. Mol Cell 39: 100-109, 2010.

29. Buyan A, Kalli AC and Sansom MS: Multiscale Simulations Suggest a Mechanism for the Association of the Dok7 PH Domain with PIP-Containing Membranes. PLOS Comput Biol 12: e1005028, 2016.

30. Chen QY and Costa M: PI3K/Akt/mTOR Signaling Pathway and the Biphasic Effect of Arsenic in Carcinogenesis. Mol Pharmacol 94: 784-792, 2018.

31. Shi H, Pu J, Zhou XL, Ning YY and Bai C: Silencing long non-coding RNA ROR improves sensitivity of non-small-cell lung cancer to cisplatin resistance by inhibiting PI3K/Akt/mTOR signaling pathway. Tumour Biol 39: 1010428317697568, 2017.

32. Zhu J, Yao J, Huang R, Wang Y, Jia M and Huang Y: Ghrelin promotes human non-small cell lung cancer A549 cell proliferation through PI3K/Akt/mTOR/P70S6K and ERK signaling pathways. Biochem Biophys Res Commun 498: 616-620, 2018

33. Lv X, Li CY, Han P and Xu XY: MicroRNA-520a-3p inhibits cell growth and metastasis of non-small cell lung cancer through PI3K/AKT/mTOR signaling pathway. Eur Rev Med Pharmacol Sci 22: 2321-2327, 2018.

34. Cui S, Wang J, Wu Q, Qian J, Yang C and Bo P: Genistein inhibits the growth and regulates the migration and invasion abilities of melanoma cells via the FAK/paxillin and MAPK pathways. Oncotarget 8: 21674-21691, 2017.

35. Du T, Qu Y, Li J, Li H, Su L, Zhou Q, Yan M, Li C, Zhu Z and Liu B: Maternal embryonic leucine zipper kinase enhances gastric cancer progression via the FAK/Paxillin pathway. Mol Cancer 13: 100, 2014.

36. Lu XS, Sun W, Ge CY, Zhang WZ and Fan YZ: Contribution of the PI3K/MMPs/Ln-5 $\gamma 2$ and EphA2/FAK/Paxillin signaling pathways to tumor growth and vasculogenic mimicry of gallbladder carcinomas. Int J Oncol 42: 2103-2115, 2013.

37. Hu YL, Lu S, Szeto KW, Sun J, Wang Y,Lasheras JC and Chien S: FAK and paxillin dynamics at focal adhesions in the protrusions of migrating cells. Sci Rep 4: 6024, 2014. 